\title{
Sind Ärzte noch die Experten in der Medizin?
}

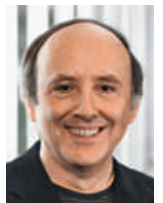

Heinz

Bonfadelli

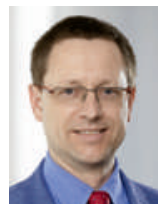

Christoph Bosshard

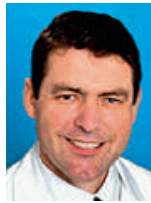

Daniel

Candinas

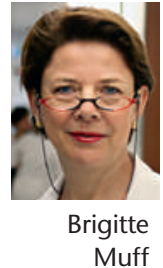

In allen Lebensbereichen und auf allen Ebenen wird nach Experten gerufen, und deren Ratschläge füllen unsere Talkshows, Websites, Zeitungen und Bücherregale. Dies betrifft insbesondere auch die Medizin. Doch gewähren diese Urteile selten die gewünschte Eindeutigkeit und Sicherheit. $\mathrm{Zu}$ widersprüchlich sind die Aussagen der selbst- oder fremdernannten Experten, zu unklar die Anforderungen an den Expertenstatus, zu stark der Wunsch nach einer Demokratisierung der Expertise.

Dies betrifft ganz speziell die Ärzteschaft. Kann und will sie überhaupt noch einen eindeutigen Expertenrat geben? Wie verhält sie sich zu den Expertisen, die in anderen Medizinalberufen und in den neuen Medien wachsen?

\section{Diskutieren Sie mit}

Die Podiumsdiskussion geht der Frage nach, ob wir in einer Krise der Expertise stehen. Woher kommt das Bedürfnis nach vielen Ratgebern? Wie gehen Ärzte damit um? Wer gilt als Experte? Wie positioniert sich die Universitätsmedizin? Gibt es einen Verteilkampf um Aufmerksamkeit? Welches ist die Rolle der Medien?

Mit dem Berner Podium möchten die Schweizerische Ärztezeitung und das Institut für Medizingeschichte der Universität Bern zu einer fundierten Auseinandersetzung mit diesen und weiteren Fragen rund um das Thema der medizinischen Expertise und den Expertenstatus von Medizinern beitragen. Der Einbezug des Publikums in die Diskussion ist zentraler Bestandteil des Konzepts der SÄZ-Podiumsveranstaltungen.

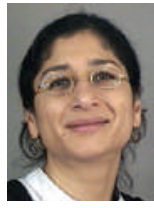

Maya

Shaha

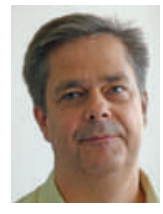

Eberhard Wolff

\section{Podiumsgäste}

Auf dem Podium diskutieren unter Leitung von SÄZ-Redaktor PD Dr. rer. soc. Eberhard Wolff:

- Prof. Dr. Heinz Bonfadelli, Institut für Publizistikwissenschaft und Medienforschung, Universität Zürich

- Dr. med. Christoph Bosshard, Mitglied Zentralvorstand der FMH, Facharzt für Orthopädische Chirurgie und Traumatologie des Bewegungapparates

- Prof. Dr. med. Dr. h. c. Daniel Candinas, Klinikdirektor Universitätsklinik für Viszerale Chirurgie und Medizin, Bereich Viszeralchirurgie, Inselspital Universität Bern

\section{Datum / Zeit / Ort}

Die Podiumsveranstaltung mit anschliessendem Apéro findet statt am Donnerstag, 5. September 2013, 18.30-20.30 Uhr, im Hörsaal 3 (Medizin, Sahli), Eingang 34 (Operationstrakt Ost), Inselspital, Universitätsspital Bern.

\section{Anmeldung}

Der Eintritt zur Veranstaltung ist frei, eine Anmeldung ist aber erforderlich. Anmeldungen können bis Dienstag, den 3. September 2013, via E-Mail an redaktion.saez[at]emh.ch oder via Fax an 061467 8556 erfolgen. Bitte Ihren Namen und die Namen allfälliger Begleitpersonen sowie das Stichwort «Anmeldung zum SÄZ-Podium vom 5. September» angeben. Auch telefonische Anmeldungen sind vormittags unter 0614678572 möglich.

\section{Veranstaltungspartner}

Die Podiumsdiskussion wird in Zusammenarbeit mit dem Institut für Medizingeschichte der Universität Bern im Rahmen der Jahrestagung der Schweizer Medizinhistoriker (SGGMN) zum Thema «Medizinische Expertise im 20. und 21. Jahrhundert» (5.-7. September, www.sggmn.ch) organisiert. Die Durchführung des Anlasses wird möglich dank grosszügiger Unterstützung durch Interpharma, den Verband der forschenden pharmazeutischen Industrie. Die Verantwortung für Konzept und Inhalt des Podiums liegt ausschliesslich bei der Schweizerischen Ärztezeitung.

- Dr. med. Brigitte Muff, Vorstandsmitglied Schweizerisches Institut für ärztliche Weiterund Fortbildung SIWF, Chefärztin Chirurgie, Spital Bülach

- Maya Shaha, PhD, RN, Wiss. Mitarbeiterin, Direktion Pflege, MTT, Universitätsspital Bern; MER, Institut universitaire de formation et de recherche en soins, Universität Lausanne 